\title{
What are the benefits of two-dimensional speckle tracking echocardiography for diagnosis and treatment follow-up of childhood-onset systemic lupus erythematosus myocarditis?
}

\author{
Gabriela Nunes leal ${ }^{1 *}$, Maria de Fátima Diniz ${ }^{1}$, Juliana Brunelli², Alessandro C. Lianza ${ }^{1}$, Adriana M. E. Sallum², \\ CLOVIS A. SILVA ${ }^{2}$
}

${ }^{1}$ Echocardiography Service, Instituto da Criança, Hospital das Clínicas, Faculdade de Medicina, Universidade de São Paulo (HC-FMUSP), São Paulo, SP, Brazil

${ }^{2}$ Pediatric Rheumatology Unit, Instituto da Criança, HC-FMUSP, São Paulo, SP, Brazil

Study conducted at the Echocardiography Service and at Pediatric Rheumatology Unit, Instituto da Criança, Hospital das Clínicas, Faculdade de Medicina, Universidade de São Paulo (HC-FMUSP), São Paulo, SP, Brazil

Article received: 6/29/2016

Accepted for publication: $7 / 26 / 2016$

*Correspondence:

Address: Av. Dr. Enéas de Carvalho Aguiar, 647

São Paulo, SP - Brazil

Postal code: 05403-000

gnleal@gmail.com

http://dx.doi.org/10.1590/1806-9282.62.06.490

Financial support: Supported by grants from Conselho Nacional de Desenvolvimento Científico e Tecnológico

(CNPq 472155/2012-1 and 303422/2015-7 - 1A to CAS), Federico Foundation to CAS and by Núcleo de Apoio à Pesquisa

"Saúde da Criança e do Adolescente" / USP (NAP-CriAd) to CAS.

\section{Case report}

A 17-year-old white female had a previous diagnosis of childhood-onset systemic lupus erythematosus (c-SLE) based on American College of Rheumatology classification criteria: arthritis, pericarditis, proteinuria $>0.5 \mathrm{~g} /$ day, and the presence of antinuclear (ANA), double-stranded DNA (ds-DNA) and antiphospholipid autoantibodies. ${ }^{1}$ She presented to the emergency room with a one-day history of fever $\left(38^{\circ} \mathrm{C}\right)$, shortness of breath, chest pain on inspiration, and orthopnea. Regarding classic cardiovascular risk factors, there was no history of arterial hypertension or chronic renal failure. Her lipid profile was considered normal for age and sex and her body mass index was $27.5 \mathrm{~kg} / \mathrm{m}^{2}$ (between $90^{\text {th }}$ and $95^{\text {th }}$ percentile). Previously to emergency room admission, she had never shown signs or symptoms of heart failure, and left ventricle (LV) ejection fraction (EF) documented by a routine echocardiogram one month earlier was $66 \%$ (normal $\geq 55 \%$, by Teichholz method). There was no chamber enlargement, ventricular hypertrophy or signs of diastolic dysfunction. Despite that, a concomitant deformation analysis of LV by two-dimensional speckle tracking echocardiography (2DST) identified reduced longitudinal peak systolic strain in the apical 4-chamber view: -15.2\%; normal range -22.18\% \pm 3.06 (My Lab 60 - Esaote, Florence, Italy). ${ }^{2}$ Of note, $\mathrm{LV}$ circumferential peak systolic strain in the mid cavity was still preserved: $-25.7 \%$ (normal range $-24 \% \pm 6){ }^{2}$ At that time, the disease activity parameter (SLE Disease Activity Index 2000 - SLEDAI-2K) ${ }^{3}$ was 18. At the emergency room, physical examination revealed fever $\left(38.5^{\circ} \mathrm{C}\right)$, tachypnea (respiratory rate of $40 / \mathrm{min}$ ), tachycardia (heart rate of $150 / \mathrm{min}$ ), cool extremities, delayed capillary filling time (5 sec), arterial hypotension $(70 \times 40 \mathrm{mmHg})$, and inspiratory crackles. Chest X-ray showed slightly enlarged cardiac silhouette and pulmonary edema. A bedside echocardiogram detected a small pericardial effusion and reduced ejection fraction (52\%), with diffuse hypocontractility of the LV. C-reactive protein was $190 \mathrm{mg} / \mathrm{L}$ (normal range < 5), erythrocyte sedimentation rate of $61 \mathrm{~mm} \mathrm{1st} / \mathrm{h}$ (normal < 20), troponin $0.046 \mathrm{ng} /$ $\mathrm{mL}$ (normal < 0.014), and ds-DNA antibodies $>200 \mathrm{IU} / \mathrm{mL}$ (normal <20). Complement system components were low: C4: $8.3 \mathrm{mg} / \mathrm{dL}$ (normal range 10-40) and C3: $71 \mathrm{mg} / \mathrm{dL}$ (normal range $90-180 \mathrm{mg} / \mathrm{dL}$ ). Both serum urea and creatinine were slightly elevated (urea $41 \mathrm{mg} / \mathrm{dL}$; normal range $11-38.5 \mathrm{mg} / \mathrm{dL}$ and creatinine $1.02 \mathrm{mg} / \mathrm{dL}$; normal range 0.5-0.9 mg/dL). Hemoglobin was $7.2 \mathrm{~g} / \mathrm{dL}$, leukocytes count was $5,880 / \mathrm{mm},{ }^{3}$ and platelets count was $408 \mathrm{~K} / \mu \mathrm{L}$. Urinary casts were present. Blood and urine cultures were negative. At admission, SLEDAI-2K was 20. A lupus flare with myocarditis was diagnosed and she was promptly sent to pediatric intensive care unit (ICU), where oxygen supply, inotropic support (milrinone) and endovenous diuretics were 
initiated. However, it was only after receiving $1 \mathrm{~g}$ of methylprednisolone for three consecutive days that heart failure symptoms disappeared and a normal LV ejection fraction was achieved (60\%). She was discharged from the pediatric ICU on day 17 and admitted to the pediatric ward with a prescription of hydroxychloroquine $400 \mathrm{mg}$ /day, prednisone $40 \mathrm{mg} /$ day, and furosemide $20 \mathrm{mg} /$ day. Another echocardiogram with 2DST was obtained on day 23, which revealed normal LV ejection fraction (65\%) but still a striking reduced longitudinal peak systolic strain in apical 4 chamber view (-9\%). Circumferential peak systolic strain in mid cavity was also below acceptable values (-16.8\%). On day 24, she presented tachypnea, basal crept, and hepatomegaly. Glasgow score was reduced (11) and endotracheal intubation was necessary. The patient was sent back to pediatric ICU and inotropic support was reintroduced. A bedside echocardiogram showed LV ejection fraction of $38 \%$ and a small pericardial effusion. By that time, SLEDAI-2K was 30. Again, it was only after a threeday pulse of methylprednisolone that a normal LV ejection fraction $(58 \%)$ was achieved. Computed tomography scan ruled out the hypothesis of pulmonary embolism or brain hemorrhage. Cerebral fluid analysis showed aseptic meningitis, suggesting neuropsychiatric lupus manifestation. She was progressively weaned from mechanical ventilation and inotropes, and was sent back to the pediatric ward on day 36. A final echocardiogram with 2DST on day 40 showed that, although LV ejection fraction was $58 \%$ and she had no more signs or symptoms of heart failure, longitudinal peak systolic strain in apical 4-chamber view was still depressed (-15.4\%). Circumferential peak systolic strain in mid cavity was once more within normal limits (-19.84\%). Intravenous cyclophosphamide infusion $\left(1 \mathrm{~g} / \mathrm{m}^{2}\right)$ was pre- scribed and she was finally sent home after 42 days of hospitalization, with a prescription of captopril $100 \mathrm{mg} /$ day, furosemide $20 \mathrm{mg}$ /day, spironolactone $50 \mathrm{mg} /$ day, prednisone $40 \mathrm{mg} /$ day, and carvedilol $75 \mathrm{mg} /$ day. LV ejection fraction throughout hospitalization is shown in Figure 1 and 2DST strain analysis in Figure 2. The Ethics Committee of our University Hospital approved this study.

\section{Discussion}

We reported here the first case of c-SLE myocarditis, in which 2DST deformation analysis was prospectively able to detect LV systolic function impairment even before the development of congestive heart failure or decreased ejection fraction. Additionally, this novel technique was clearly helpful in decision making throughout treatment.

Myocarditis is a well-known life threatening complication of c-SLE and may present itself as an acute illness or have a chronic course, due to myocardial inflammation, small vessel vasculitis and interstitial fibrosis. ${ }^{4} \mathrm{Di}$ agnosis is usually based on clinical findings of congestive heart failure, elevated cardiac enzymes, and decreased LV ejection fraction on echocardiogram. Other comorbidities also contribute to myocardial dysfunction in c-SLE and should always be ruled out, such as systemic hypertension, dyslipidemia, obesity, and chronic renal failure. ${ }^{5}$ Magnetic resonance imaging may support the diagnosis of myocarditis, although it is limited by poor availability on emergency basis. Endomyocardial biopsy is reserved for doubtful cases, owing to its invasive nature and procedure related risks.

Of note, 2DST is an angle-independent method for echocardiographic assessment of myocardial deformation in longitudinal, radial and circumferential directions,

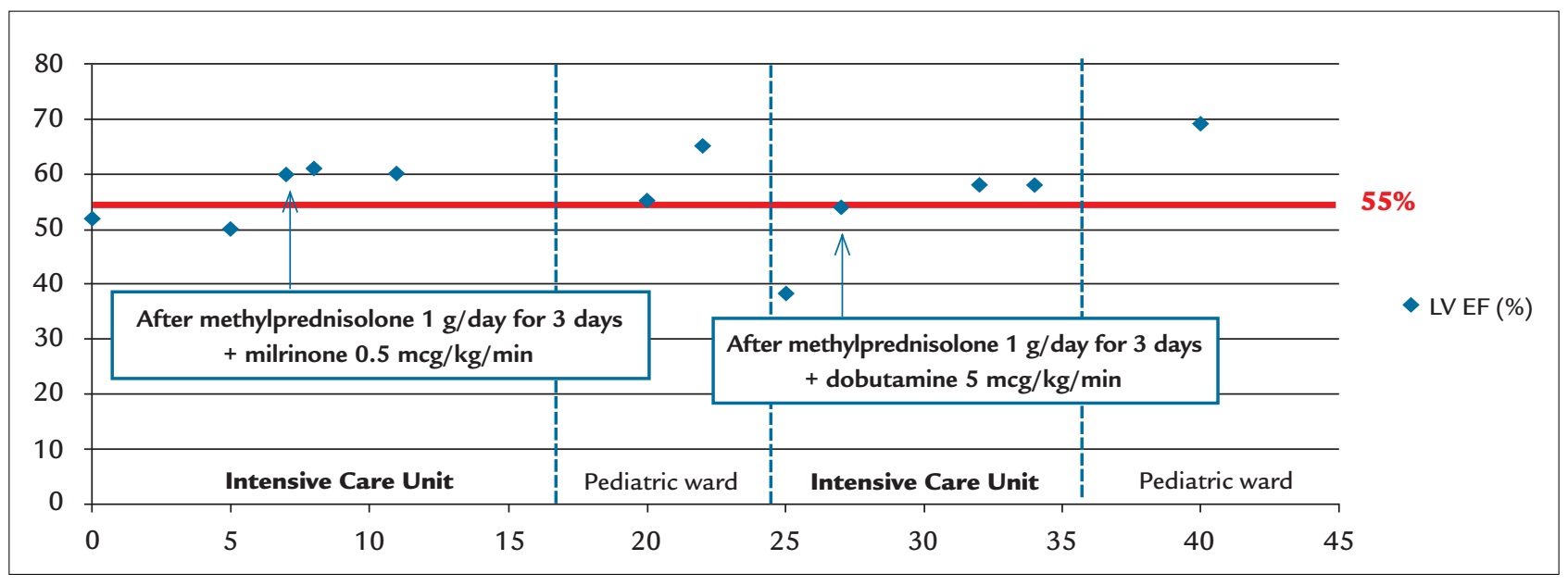

FIGURE 1 Left ventricle (LV) ejection fraction (EF) vs. days from admission. 


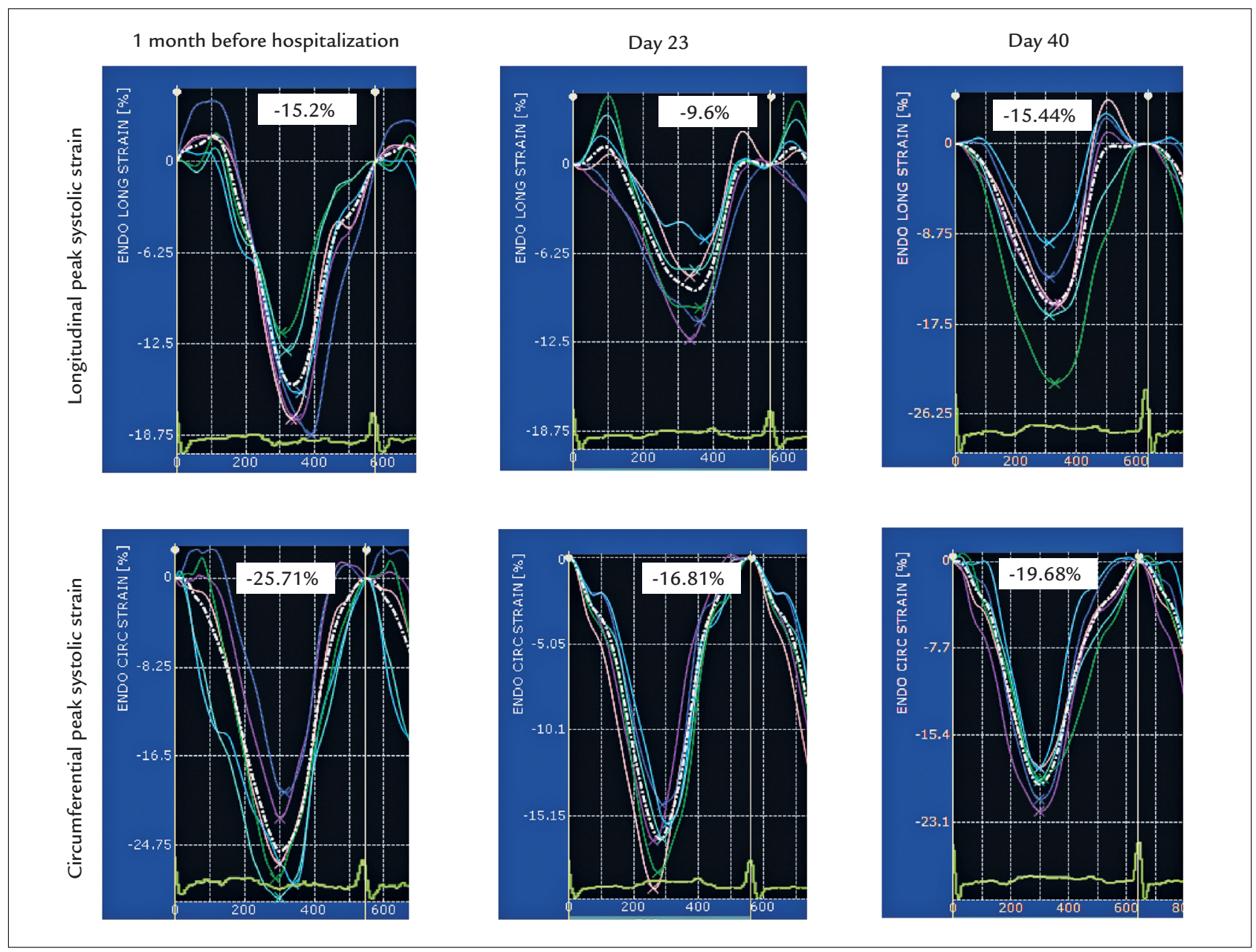

FIGURE 2 Longitudinal and circumferential peak systolic strain.

and was recently considered useful in two cross-sectional studies as a tool to investigate c-SLE patients. ${ }^{6,7}$ 2DST deformation analysis has also been proven as a valuable echocardiographic tool for detection of subclinical myocardial compromise in a wide range of diseases, with undoubted prognostic and therapeutic implications. ${ }^{8} \mathrm{Al}-$ though negative correlation between SLEDAI-2K and LV longitudinal peak systolic strain has already been documented by transversal studies in c-SLE patients with no signs or symptoms of heart failure, ${ }^{7}$ no prospective study has ever documented LV myocardial deformation pattern of patients throughout as well as in-between flares.

Longitudinal mechanics, which relies on subendocardial fibers, is the most vulnerable and sensitive to the presence of myocardial aggression, thus being affected first. Circumferential mechanics, resultant of mid-myocardial layers deformation, are the last to be affected in the progression of diseases and usually maintains LV pump and guarantees a preserved ejection fraction in a subclinical stage. ${ }^{9}$ This explains why the routine echocardiogram performed one month before emergency room admission of our patient showed reduced longitudinal strain, albeit preserved circumferential strain and ejection fraction. Indeed, as inflammation status progressed, a huge decrease of $\mathrm{LV}$ longitudinal and circumferential strain preceded ejection fraction reduction and her second intensive care unit admission. In face of such troublesome evolution, although she eventually experienced recovery of LV ejection fraction and was completely asymptomatic at the time of discharge, the still impaired longitudinal strain motivated clinicians to prescribe additional intravenous cyclophosphamide, as well as to maintain heart failure therapy at home, adding carvedilol to the therapeutic arsenal.

SLE is a systemic disease with simultaneous involvement of various organs and systems. ${ }^{10}$ Treatment options for severe lupus manifestations, including myocarditis, 
may include high-dose glucocorticoids, intravenous cyclophosphamide, and intravenous immunoglobulin, ${ }^{11}$ even though there is no consensus on the most appropriate way to manage those patients. We wonder if the introduction of carvedilol by the time longitudinal strain compromise was first detected by a routine echocardiogram could have altered the clinical course in this particular case. In fact, carvedilol antioxidant properties were associated with inflammatory cytokines suppression in experimental autoimmune myocarditis. ${ }^{12}$

Prospective multicenter studies using 2DST myocardial deformation analysis should be conducted in order to establish the ideal moment to address specific treatment for lupus myocarditis, given that myocardial compromise contributes considerably for morbidity and mortality among c-SLE patients. We hypothesize that better cardiovascular outcome in future life may be achieved with earlier myocarditis diagnosis and management in cSLE patients.

\section{References}

1. Hochberg MC. Updating the American college of rheumatology revised criteria for the classification of systemic lupus erythematosus. Arthritis Rheum. 1997; 40(9):1725.

2. Bussadori C, Moreo A, Di Donato M, De Chiara B, Negura D, Dall'Aglio E, et al. A new 2D-based method for myocardial velocity strain and strain rate quantification in a normal adult and paediatric population: assessment of reference values. Cardiovasc Ultrasound. 2009; 7:8.
3. Gladman DD, Ibañez D, Urowitz MB. Systemic lupus erythematosus disease activity index 2000. J Rheumatol. 2002; 29(2):288-91.

4. Apte M, McGwin G Jr, Vilá LM, Kaslow RA, Alarcón GS, Reveille JD; LUMINA Study Group. Associated factors and impact of myocarditis in patients with SLE from LUMINA, a multiethnic US cohort. Rheumatology (Oxford) 2008; 47(3):362-7.

5. Zawadowski GM, Klarich KW, Moder KG, Edwards WD, Cooper LT Jr. A contemporary case series of lupus myocarditis. Lupus. 2012; 21(13):1378-84.

6. Leal GN, Silva KF, França CM, Lianza AC, Andrade JL, Campos LM, et al. Subclinical right ventricle systolic dysfunction in childhood-onset systemic lupus erythematosus: insights from two-dimensional speckle-tracking echocardiography. Lupus. 2015; 24(6):613-20.

7. Leal GN, Silva KF, Lianza AC, Giacomin MF, Andrade JL, Kozu K, et al. Subclinical left ventricular dysfunction in childhood-onset systemic lupus erythematosus: a two-dimensional speckle-tracking echocardiographic study. Scand J Rheumatol. 2016; 45(3):202-9.

8. Russo C, Jin Z, Elkind MS, Rundek T, Homma S, Sacco RL, et al. Prevalence and prognostic value of subclinical left ventricular systolic dysfunction by global longitudinal strain in a community based cohort. Eur J Heart Fail. 2014; 16(12):1301-9.

9. Mor-Avi V, Lang RM, Badano LP, Belohlavek M, Cardim NM, Derumeaux $\mathrm{G}$, et al. Current and evolving echocardiographic techniques for the quantitative evaluation of cardiac mechanics: ASE/ EAE consensus statement on methodology and indications endorsed by the Japanese Society of Echocardiography. J Am Soc Echocardiogr. 2011; 24(3):277-313.

10. Gomes RC, Silva MF, Kozu K, Bonfá E, Pereira RM, Terreri MT, et al. Features of 847 childhood-onset systemic lupus erythematousus patients in three age groups at diagnosis: a Brazilian multicenter study. Arthritis Care Res (Hoboken). 2016 (in press).

11. Silva CA, Aikawa NE, Pereira RM, Campos LM. Management considerations for childhood-onset systemic lupus erythematosus patients and implications on therapy. Expert Rev Clin Immunol. 2016; 12(13):301-13.

12. Yuan Z, Shioji K, Kihara Y, Takenaka H, Onozawa Y, Kishimoto C. Cardioprotective effects of carvedilol on acute autoimmune myocarditis: anti-inflammatory effects associated with antioxidant property. Am J Physiol Heart Circ Physiol. 2004; 286(1):H83-90. 